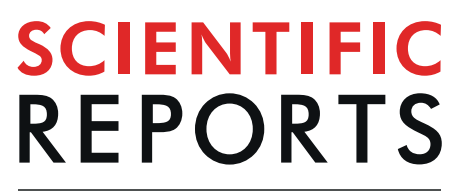

\title{
Early symptoms and sensations as predictors of lung cancer: a machine learning multivariate model
}

\author{
Adrian Levitsky $\mathbb{1 0}^{1,2}$, Maria Pernemalm $\mathbb{1}^{2}{ }^{2}$, Britt-Marie Bernhardson ${ }^{1}$, Jenny Forshed ${ }^{2}$ \\ Karl Kölbeck ${ }^{3}$, Maria Olin ${ }^{3}$, Roger Henriksson ${ }^{4}$, Janne Lehtiö $\mathbb{D}^{2}{ }^{2}$, Carol Tishelman ${ }^{1,5,6}$ \& \\ Lars E. Eriksson $\mathbb{B}^{1,7,8^{*}}$
}

The aim of this study was to identify a combination of early predictive symptoms/sensations attributable to primary lung cancer (LC). An interactive e-questionnaire comprised of pre-diagnostic descriptors of first symptoms/sensations was administered to patients referred for suspected LC. Respondents were included in the present analysis only if they later received a primary LC diagnosis or had no cancer; and inclusion of each descriptor required $\geq 4$ observations. Fully-completed data from $506 / 670$ individuals later diagnosed with primary LC $(n=311)$ or no cancer $(n=195)$ were modelled with orthogonal projections to latent structures (OPLS). After analysing 145/285 descriptors, meeting inclusion criteria, through randomised seven-fold cross-validation (six-fold training set: $n=433$; test set: $n=73), 63$ provided best LC prediction. The most-significant LC-positive descriptors included a cough that varied over the day, back pain/aches/discomfort, early satiety, appetite loss, and having less strength. Upon combining the descriptors with the background variables current smoking, a cold/flu or pneumonia within the past two years, female sex, older age, a history of COPD (positive LC-association); antibiotics within the past two years, and a history of pneumonia (negative LCassociation); the resulting 70-variable model had accurate cross-validated test set performance: area under the ROC curve $=0.767$ (descriptors only: $0.736 /$ background predictors only: 0.652 ), sensitivity $=84.8 \%(73.9 / 76.1 \%$, respectively $)$, specificity $=55.6 \%(66.7 / 51.9 \%$, respectively). In conclusion, accurate prediction of LC was found through 63 early symptoms/sensations and seven background factors. Further research and precision in this model may lead to a tool for referral and LC diagnostic decision-making.

Lung cancer (LC) remains the leading cause of cancer-related mortality ${ }^{1-3}$. While LC generally manifests with early symptoms and sensations, they are often so diffuse that care-seeking may be delayed ${ }^{4,5}$. Traditional risk factors, i.e. smoking, are not optimal in discriminating LC due to poor model performance ${ }^{6,7}$, thus, keen general practitioner vigilance ${ }^{8-10}$ and quick access to sensitive screening tools are needed ${ }^{10-12}$. While low-dose computerised tomography has been shown to be an important screening tool for $\mathrm{LC}^{13,14}$, it also suffers a high false-positive rate ${ }^{13-15}$ and should only be applied for particular risk groups. Thus, the need to identify early risk symptoms and sensations of LC that can flag individuals for screening and early detection remains ${ }^{9,10}$; this can be achieved from in-depth early symptomatic investigations.

Earlier identification of LC symptoms and sensations would have a major impact on overall LC mortality due to profoundly greater survival in early-identified stages ${ }^{16}$. Large cohort investigations from diffuse general

\footnotetext{
${ }^{1}$ Division of Innovative Care Research, Department of Learning, Informatics, Management and Ethics (LIME), Karolinska Institutet, SE-171 77, Solna, Sweden. ${ }^{2}$ Cancer Proteomics Mass Spectrometry, Department of OncologyPathology, Karolinska Institutet, Science for Life Laboratory, SE-171 65, Solna, Sweden. ${ }^{3}$ Lung Oncology Center, Cancer Theme, Karolinska University Hospital, SE-171 76, Solna, Sweden. ${ }^{4}$ Department of Radiation Sciences and Oncology, University of Umeå, SE-901 87, Umeå, Sweden. ${ }^{5}$ Center for Health Economy, Informatics and Health System Research (CHIS), Stockholm Health Care Services (SLSO), Region Stockholm, SE-113 65, Stockholm, Sweden. ${ }^{6}$ The Centre for Rural Medicine (Glesbygdsmedicinskt Centrum GMC), Region Västerbotten, SE-923 31 , Storuman, Sweden. ${ }^{7}$ School of Health Sciences, City, University of London, Northampton Square, London, EC1V $\mathrm{OHB}$, United Kingdom. ${ }^{8}$ Department of Infectious Diseases, Karolinska University Hospital, SE-141 86, Huddinge, Sweden. *email: lars.eriksson@ki.se
} 
practice medical records have thus far uncovered some LC-risk signs and symptoms, e.g. haemoptysis, dyspnoea, chest pain, cough, appetite loss and/or weight loss up to two years before diagnosis ${ }^{17-20}$. Only one prospective study $^{21}$, to our knowledge, evaluated a symptom survey administered to patients referred for LC investigation before the individuals met a specialist or had received any primary LC diagnosis. Haemoptysis was a possible LC predictor, although only twenty descriptors were investigated ${ }^{21}$. A driving need thus remains for identifying a combination of pre-diagnostic individual descriptors that can predict primary LC.

Study aim. This study was conducted to fill the gap left by limited investigations of patient-reported pre-diagnostic LC descriptors, contributing a more thorough investigation of patient experiences. The aim of this study is thus to identify a combination of early predictive symptoms and sensations attributable to LC.

\section{Methods}

Study conduction and sample. After approval by the Stockholm regional ethics board (EPN: ref no 2014/1290-32), data was collected from September 2014-November 2015. In Stockholm County, diagnostic work-up for suspected LC is centralised to Karolinska University Hospital (KUH). Thus, all consecutive patients referred to KUH were asked to participate in the study and sent written study information before their first scheduled visit. Upon the first visit, written informed consent was obtained. Patients then completed the Patient EXperience of Bodily Changes for Lung Cancer Investigation (PEX-LC) e-questionnaire on a touch screen user interface on a smart tablet directly before their clinical visit with a pulmonary medicine physician. Research assistants were available for help. Medical records of eventual diagnosis were later retrieved, with a follow-up of at least one year after questionnaire completion. This study was carried out according to the Declaration of Helsinki and data were anonymized to protect the privacy of the study participants.

The PEX-LC instrument. The PEX-LC instrument is an e-questionnaire focusing on patients' own specific pre-diagnostic descriptions of early symptoms or sensations, hereafter referred to as descriptors. The instrument was derived from prior qualitative interviews $(n=60)$ conducted at several Swedish lung medicine departments. PEX-LC consists of 11 individualised, interactive modules on a touch screen smart tablet: Background (e.g. sociodemographic characteristics, comorbidities and smoking habits), Breathing Difficulties, Cough, Phlegm/ Expectorates, Pain/Aches/Discomfort, Fatigue, Voice Changes, Appetite/Eating/Taste Changes, Olfactory Changes, Fever/Chills/Sweating, and Other Changes (e.g. general physical condition, malaise, or other emotional changes). There are 342 potential items; 285 descriptors indicative of the first symptoms/sensations the patient noticed that had caused a change in their lives, and 57 background variables. Patient-reported recall of early descriptors is recorded in binary form ("yes"/"no"). PEX-LC was tailored to allow each individual participant to complete only those items appropriate for the specific individual's onset of symptoms or sensations.

Statistical analyses. Descriptors and background variables meeting inclusion criteria ( $\geq 4$ observations for LC and for no cancer (NC), respectively (software default, SIMCA v.14.1)) were first analysed by principal component analysis (PCA) for data inspection for potential biases in the data, such as clusters or outliers which could skew findings ${ }^{22}$. Orthogonal projections to latent structures (OPLS) discriminant analysis (detailed description below) with cross-validation (CV) was then carried out to class-separate the data between the predicted (LC vs. NC) and orthogonal (structured noise) states ${ }^{23-26}$ (SIMCA v.14.1). Univariate associations to LC were analysed with binary logistic regression, and proportional (e.g. gender) and continuous data (age) were analysed with Pearson's chi-squared tests and Independent Samples Mann-Whitney U tests, respectively (IBM SPSS v.24).

Orthogonal projections to latent structures (OPLS) discriminant analysis. An OPLS modelling approach was utilised to analyse variables (descriptors) covarying with outcome (LC or NC) $)^{23-26}$. Analyses were performed with SIMCA v.14.1, Umetrics ${ }^{\mathrm{TM}}$ Suite, Sartorius Stedim Biotech. Inclusion criteria were full-module completion (no missing data) and $\geq 4$ observations for descriptors, and a diagnosis of primary LC or NC (other cancer diagnoses led to exclusion) for patients.

Cross-validation estimates the predictive performance of a model, thus ensuring model reliability. Applying CV with OPLS in SIMCA avoids model overfitting by only retaining significant components in the model ${ }^{27}$. $K$-fold CV was carried out with $1 / 7^{\text {th }}$ of the dataset being excluded for each round (software default ${ }^{28}$ ) up until and including the sixth group (six-fold CV for the training set). The seventh group was the CV test set, independent of model training.

To ensure cohort representativeness and to remove any potential bias created by chance due to row placement $^{27}$, all seven CV groups were created by block-randomisation to have similar proportions of LC ( $\left.60 \%\right)$ vs. $\mathrm{NC}(\sim 40 \%)$ as expressed in the entire dataset, in addition to randomised row placement. This block-randomisation also took full dataset representativeness of LC histology (Fig. 1) into consideration (non-small cell, 80-85\% vs. small cell/other, $15-20 \%$ for each of the seven groups).

Model selection. Multivariate regression models through OPLS were created through selection from key criteria, including PCA loadings for background variables, OPLS projection loadings, explained variance, and sensitivity over specificity, listed as follows. The first model included potential LC-associated background variables and descriptors meeting inclusion criteria, which served as the basis for all models as it projected all variables' relative importance for overall model contribution. The theoretical foundation of PLS/OPLS is that it is hypothetically more precise with a higher load of potential variance-explaining variables from multi-dimensional interactions ${ }^{28}$. Variables were thus excluded sequentially through visual inspection of OPLS regression coefficients (which reflect each variable's importance in relation to the first (predictive) component) as well as through inspection of variable importance for the projection (VIP) values (which indicate overall model contribution, both to prediction and to structured noise). Maximal explained variance of LC within the training set $\left(\mathrm{R}^{2}\right)$ and 


\section{CONSORT Flow Diagram}

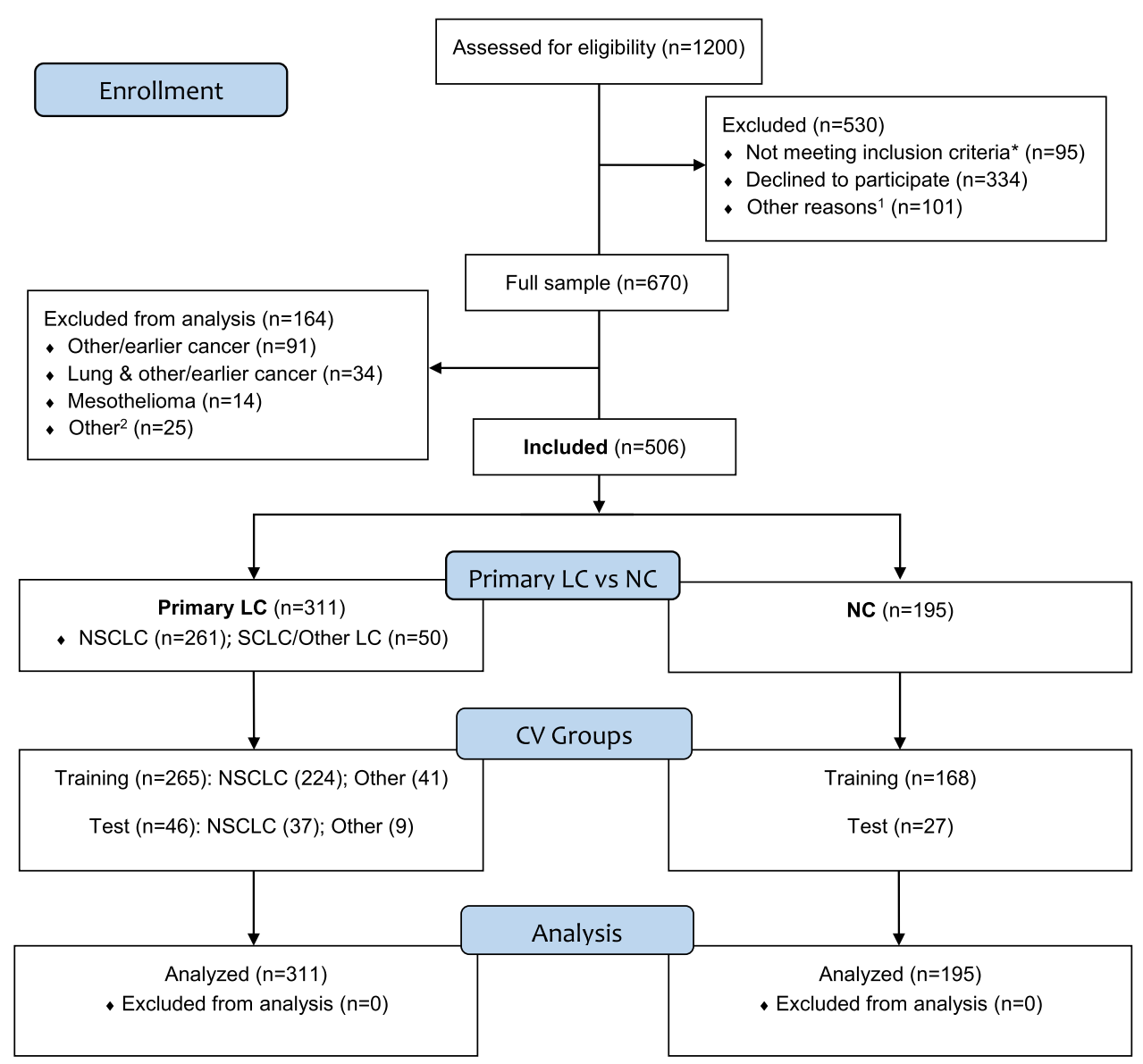

Figure 1. CONSORT flow diagram: The PEX-LC lung cancer investigation cohort. This figure is based on the CONSORT 2010 flow diagram. As this was not a randomised intervention trial, it has been modified to suit this cohort study accordingly. Primary LC: primary lung cancer (no other cancer); NC, no cancer; NSCLC: non-small cell lung cancer (adenocarcinoma, $n=200$; squamous cell carcinoma, $n=45$; not otherwise specified (NOS), $n=5$; other NSCLC (adenosquamous lung carcinoma $(n=4)$, large cell neuroendocrine carcinoma $(\mathrm{n}=3)$; large cell carcinoma, adenoid cystic carcinoma of the lung, adenoid carcinoma with neuroendocrine differentiation, and mucoepidermoid carcinoma of the lung $(n=1$, respectively)); SCLC: Small cell lung cancer (includes one individual with combined SCLC) $(\mathrm{n}=24)$; Other LC: carcinoid, $\mathrm{n}=9$; no histology, $\mathrm{n}=17$. *Not meeting inclusion criteria: translator required $(n=50)$, consent withdrawn/missing $(n=15)$; missing data $(n=5)$; other reason such as or pain, illness, or other medical condition $(n=25) .{ }^{1}$ Other reasons: Limited time of the visit or lack of resources (staff) at the clinic $(n=47)$; hospitalisations $(n=34)$; deaths $(n=20) .{ }^{2}$ Other: Medical records non-consent $(n=4)$; unconfirmed, possible lung cancer $(n=3)$; undiagnosed cancer $(n=2)$; death before clinical investigation $(n=1)$; participant withdrew clinical investigation $(n=2)$; previous lung cancer $(n=1)$; incomplete modules $(n=12)$. Primary LC: Current/previous comorbidities include Crohn's disease, diabetes, gout, lymphedema, pulmonary fibrosis, fibromyalgia, sarcoidosis ( $n=1$, respectively); rheumatoid arthritis $(\mathrm{n}=2)$; asbestos-related disease $(\mathrm{n}=3)$; heart disease or anaemia $(\mathrm{n}=4$, respectively); chronic bronchitis $(n=5)$; angina pectoris $(n=15)$; emphysema $(n=17)$; pulmonary oedema $(n=33)$; asthma $(n=35)$; chronic obstructive lung disease (COPD, $n=70)$; pneumonia $(n=73)$; no comorbidities/ unknown $(\mathrm{n}=113)$. NC (no malignant cancer): Diagnoses included Castleman's disease, empyema, systemic lupus erythematosus, gout, polymyositis, previous granulomatosis with polyangiitis, haemoptysis, tuberculosis $(\mathrm{n}=1$, respectively); benign hamartoma, resected benign hamartoma, tularaemia $(\mathrm{n}=2$, respectively); diabetes, sarcoidosis $(n=3$, respectively). Current/previous conditions, NC: asbestos-related disease, bronchitis, kidney failure or lung embolism $(n=1$, respectively); anaemia $(n=3)$; chronic bronchitis $(n=5)$; emphysema $(n=6)$; angina pectoris $(n=7)$; pulmonary oedema $(n=25)$; heart disease or COPD $(n=26$, respectively); asthma $(\mathrm{n}=34)$; pneumonia $(\mathrm{n}=58)$; no diagnosis/unknown $(\mathrm{n}=73)$.

CV-explained variance in the test set $\left(Q^{2} ;>50 \%\right.$, respectively - considered good predictability $\left.{ }^{27}\right)$ was the criteria for a model to be evaluated, with highest possible $\mathrm{R}^{2}$ and $\mathrm{Q}^{2}$ values being prioritised. Thus, before each sequential variable would be totally removed from a model, explained $L C$ variance $\left(R^{2}\right.$ and $\left.Q^{2}\right)$ would be cross-referenced 


\begin{tabular}{|l|c|l|l|}
\hline Variable & $\begin{array}{l}\text { Analysed } \\
(\mathbf{n = 5 0 6})^{\mathbf{a}}\end{array}$ & $\begin{array}{l}\text { Excluded } \\
(\mathbf{n = 1 6 4})^{\mathbf{a}}\end{array}$ & P value \\
\hline Age, years (Median (IQR)) & $70(63-75)$ & $72(64.3-78)$ & $\mathbf{0 . 0 0 8}$ \\
\hline Sex, females & $249(49.2)$ & $80(48.8)$ & 0.924 \\
\hline Current smokers* & $148(29.2)$ & $28(17.1)$ & $\mathbf{0 . 0 0 2}$ \\
\hline Confirmed history of asthma & $68(13.4)$ & $13(7.9)$ & 0.060 \\
\hline Confirmed history of COPD & $93(18.4)$ & $20(12.2)$ & 0.066 \\
\hline Confirmed history of pneumonia & $126(24.9)$ & $38(23.2)$ & 0.654 \\
\hline Antibiotics, past 2 years & $193(38.1)$ & $52(31.7)$ & 0.137 \\
\hline Cold/flu/pneumonia, past 2 years & $351(69.4)$ & $104(63.4)$ & 0.156 \\
\hline
\end{tabular}

Table 1. Patient characteristics in the total PEX-LC cohort. To compare patient characteristics between the individuals fulfilling study criteria (lung cancer or no cancer $=$ analysed) and the remainder of the cohort (excluded), chi-squared tests (Fisher's exact tests if expected counts $<5$ ) were utilised to compare proportional data (e.g. proportion of females or current smokers), and Independent Samples Mann-Whitney U tests

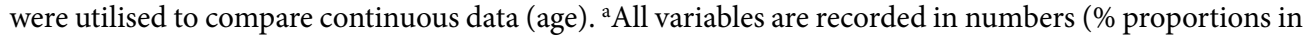
parentheses), unless specified. *Current smokers includes individuals who recently quit smoking (within the past 1 year). IQR: interquartile range; COPD: chronic obstructive pulmonary disease. History of asthma, COPD or pneumonia, respectively, are physician-confirmed. Bolded two-sided p-values $<0.050$ were considered statistically significant.

pre- and post-removal. Variables offering no model contribution were removed sequentially in this fashion. As the seven $\mathrm{CV}$ groups were always the same, to ensure that this sequential removal of variables did not overfit the model for the CV test set, 100 model simulations of randomised outcome (LC or NC) were carried out to ensure that by-chance $\mathrm{R}^{2}$ and $\mathrm{Q}^{2}$ were in all 100 instances worse than final model metrics.

The final model was chosen by selecting a cut-off with high sensitivity over specificity in the CV test set. Areas under the receiver operating characteristic (ROC) curves (AUC) for the CV test set were calculated from OPLS-generated LC prediction scores from each model, and were compared to find the most clinically-applicable model - with the maximal sensitivity over specificity ROC point by the Youden's index - in IBM SPSS v.24. Acceptable model discrimination for the test set was determined by AUC $>0.7^{29}$.

\section{Results}

Of the 1200 potentially-eligible patients investigated for suspected LC, 670 individuals agreed to participate (age and gender did not differ between those participating and the remaining potentially-eligible patients, data not shown). Of the participating patients, 506 were later diagnosed with primary LC or NC $(\mathrm{n}=311,195$, respectively); the remaining 164 patients were excluded primarily due to different/multiple diagnoses (Fig. 1). The analysed sample was marginally, although statistically significantly younger, and more often current smokers than the excluded group (basic demographics, Table 1).

PCA: Data inspection of included descriptors. A PCA was performed on 145/285 early descriptors together with $16 / 57$ background variables. The remaining variables were excluded due to not meeting inclusion criteria ( $<4$ observations in LC or NC, respectively: 140 descriptors, two background variables), or, additionally, if they were background variables that either demonstrated no univariate associations to LC, would potentially overfit the model, or were not known LC risk factors $(n=39)$ (variable selection process, Model I: Fig. 2; excluded variables: Supplementary Table S1). In the next step, 9/16 background variables were removed due to lack of explained variance (PCA loadings $<0.1$ ) or overfitting the model (Model II: Fig. 2, excluded variables: Supplementary Table S2). Thus, the next and final PCA included seven background variables (Table 2). No irregular clustering or outliers were found among individuals with LC or NC (Supplementary Fig. S1). There were no differences in individual score distributions among the PCA quadrants when having inspected for variables such as age, smoking, sex, site of enrolment, LC histology or stage, and CV group (not shown).

OPLS models and performance. The 145 descriptors were first modeled in OPLS together with the 16 background variables, which confirmed low contributions of the nine background variables removed in the PCA (OPLS VIP values $<1$ ). The next model thus included 145 descriptors and seven background variables as in the final PCA. Thereafter, a trimmed OPLS model with 70 variables was discovered through an iterative optimisation process evaluating both maximal explained LC variance as well as best prediction of LC in the CV test set (AUC > 0.7) (Table 3). In brief, the model was trimmed by sequential removal of descriptors with no model contribution (Final Model: Fig. 2; excluded variables: Supplementary Table S2). Of relevancy for this study, the largest Youden's index for sensitivity ( 0.402$)$ was selected: sensitivity $=84.8 \%$; specificity $=55.6 \%$. Figure 3 illustrates the ROC curves for the final model, indicating diagnostic model performance from predicted scores from the CV test set, including the full model with 70 variables, the 63 descriptors only, or the seven background variables only. Fig. S2A,B demonstrates the final model selection of 63/145 descriptors with seven background variables through variable count vs. explained variance. The majority of selected descriptors were from the Breathing, Cough, and Pain/Aches/Discomfort modules ( $>8$ from each, respectively) (Table 2).

All 70 variables were instrumental in maximal variance explanation and accurate LC prediction. However, should the prediction need to be centralised to one component, 14/42 positive predictors of LC were significantly 


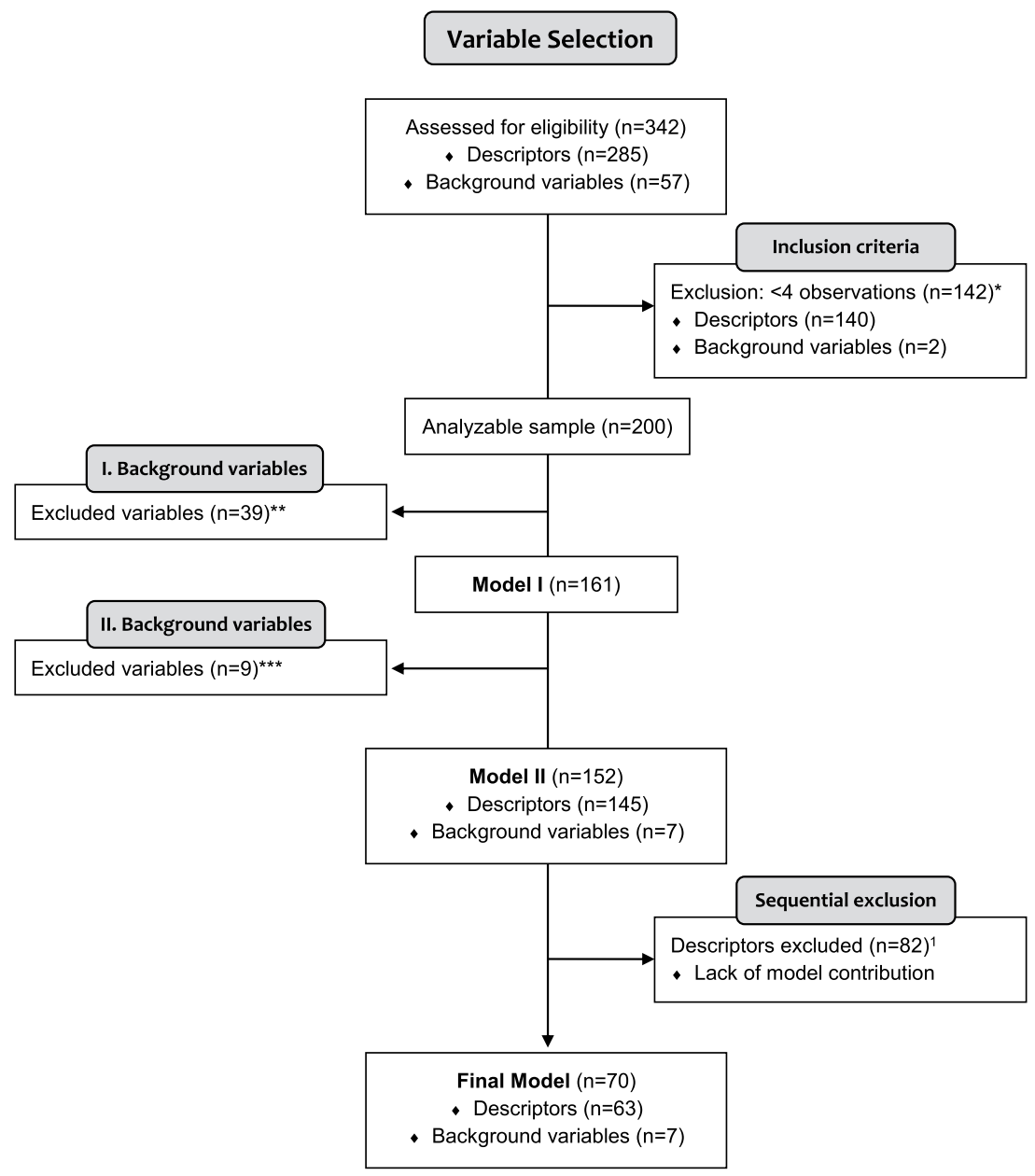

Figure 2. Variable selection flow diagram for the PEX-LC analysis. *The first exclusion step removed variables with limited observations ( $<4$ observations of "yes" per variable for each outcome: lung cancer (LC) vs. no cancer). These variables are shown in Supplementary Table S1. **For step 1 of background variable removal for potentially-analysable results, the majority were not included due to lack of significant univariate associations to LC and/or were not previously-reported LC risk signs $(n=35 / 39)$. Ordinal smoking status (never-smokers, past smokers, current smokers), living alone, and university-level education were not included due to potentially overfitting the model, and weight loss was not included due to a large proportion of missing data. These variables are shown in S1 Table. ***For step 2 of background variable removal, the majority had principal component analysis loadings and orthogonal projections to latent structures variable importance for the projection (VIP) scores $<1(n=8)$. The past smokers (vs. non-smokers) variable was not included due to the potential risk of overfitting the model, as current smokers included those who quit smoking within the past 1 year. These variables are shown in Supplementary Table S2. ${ }^{1}$ Descriptors with minimal model contribution (Supplementary Table S2) were sequentially removed $(\mathrm{n}=82)$ until maximal model performance could be achieved with 70 variables. The final model selection process including performance of additional models by variable count is shown in Supplementary Fig. S2A,B.

predictive of LC (significant descriptors bolded in Table 2; all regression coefficients: Supplementary Fig. S3), which includes, in order of magnitude, background predictors: current smoking, cold/flu/pneumonia within the past two years, female sex, and older age; and the following descriptors: a cough that varied over the day, back pain/aches/discomfort, early satiety, appetite loss, having less strength, breathing worse upon exertion, haemoptysis/hematemesis, a heightened sensitivity to different smells, consistent aches, and a voice that got more rough/ coarse. Of 28 LC-negatively-associated variables, having had antibiotics within the past two years had a significantly lower association to LC (Table 2; Supplementary Fig. S3).

The 70-variable model resulted in accurate model performance in the CV test set $(n=73)$ : area under the ROC curve $=0.767$ (descriptors only: $0.736 /$ background predictors only: 0.652 ), sensitivity $=84.8 \%(73.9 / 76.1 \%$, respectively), specificity $=55.6 \%(66.7 / 51.9 \%$, respectively $)$. As indicated in the performance parameters, the seven background predictors alone (AUC $=0.652$ ) failed to meet good diagnostic accuracy, while, upon excluding background predictors, independent LC prediction among descriptors was still demonstrated (AUC $=0.736$ ) (Table 3). OPLS scores plots and all three components for the final model training set and CV test set are shown in Fig. 4A,B, respectively, and a biplot with both scores and variable loadings in Supplementary Fig. S4. 


\begin{tabular}{|c|c|}
\hline \multicolumn{2}{|l|}{ BACKGROUND } \\
\hline Current smoking & Confirmed history of COPD \\
\hline A cold, flu or pneumonia within the past 2 years & Confirmed history of pneumonia* \\
\hline Female sex & Antibiotics within the past 2 years* \\
\hline \multicolumn{2}{|l|}{ Older age (+1 SD, unit-variance scaled age) } \\
\hline \multicolumn{2}{|l|}{ BREATHING } \\
\hline 5: Wheezing/panting* & 30: Breathing worse when I lay down* \\
\hline 7: Gasped for breath & 31: Breathing worse due to high humidity \\
\hline 12: Felt thickness in throat & 33: Breathing worse due to coldness* \\
\hline 21: Breathing sound: Whistled & 35: Breathing worse during certain times of the day* \\
\hline \multicolumn{2}{|l|}{ 29: Breathing worse upon exertion } \\
\hline \multicolumn{2}{|l|}{ COUGH } \\
\hline 3: Sudden, loud cough* & 11: Needed to clear my throat* \\
\hline 4: Hacking cough* & 29: Cough varied over the day \\
\hline 5: Wheezing cough* & 35: Cough varied over the year \\
\hline 6: Irritating, dry cough & 63: Cough occurred/worsened when I exerted myself* \\
\hline 7: Coughed until I lost my breath, choked and/or vomited* & 64: Cough occurred/worsened when I breathed deeply* \\
\hline 8: Cough attacks* & 68: Cough worsened by high humidity \\
\hline \multicolumn{2}{|l|}{ 10: Small coughs* } \\
\hline \multicolumn{2}{|l|}{ PHLEGM/EXPECTORATES } \\
\hline 3: Decreased amount* & 24: Thin, fluid-like consistency* \\
\hline 6: White mucus or sputum* & 25: Taffy-like/viscous consistency* \\
\hline \multicolumn{2}{|c|}{ 15: Haemoptysis/hematemesis (blood-mixed/brown sputum) } \\
\hline \multicolumn{2}{|l|}{ PAIN/ACHES/DISCOMFORT } \\
\hline 3: Hurting: Comes and goes & 67: Heartburn \\
\hline 8: Aches: Consistent & 201: Pain/aches/discomfort: Throat* \\
\hline 9: Aches: Comes and goes & 204: Pain/aches/discomfort: Shoulder blade \\
\hline 10_11_12: Aches: Positional/breathing-based & 207: Pain/aches/discomfort: Shoulder(s) \\
\hline 14: Pain: Consistent & 210: Pain/aches/discomfort: Neck \\
\hline 16_17_18: Pain: Positional/breathing-based* & 213: Pain/aches/discomfort: Chest \\
\hline 27: Cramping aches/pains: Comes and goes* & 223: Pain/aches/discomfort: Back \\
\hline 39: Dull aches/pain: Comes and goes & 227: Pain/aches/discomfort: Moves around* \\
\hline \multicolumn{2}{|l|}{ 49: Tenderness } \\
\hline FATIGUE & VOICE CHANGES \\
\hline 3: Less strength, got weaker & 1: Voice got more hoarse \\
\hline 4: Legs cannot cope & 2: Voice got more rough/coarse \\
\hline 11: Felt constant tiredness, weakness, or lack of energy* & 6: Cleared my throat more when I talked* \\
\hline APPETITE/EATING/TASTE CHANGES & OLFACTORY CHANGES \\
\hline 1: Appetite loss & 1: More difficult to distinguish smells \\
\hline 2: Enjoyed food less than before & 2: Lost sense of smell* \\
\hline 5: Early satiety (feeling full quicker) & 3: Heightened sensitivity to different smells \\
\hline FEVER & OTHER CHANGES \\
\hline 1: Chills* & 1: Cramps in calves \\
\hline 4: Felt cold & 10: Drier skin* \\
\hline \multirow[t]{2}{*}{ 13: Night sweats } & 13: Drier mouth \\
\hline & 19: Feeling unfit \\
\hline
\end{tabular}

Table 2. Identified descriptors and background factors for maximal lung cancer prediction performance. Variables included in the final model $(n=70)$ are shown, including 7 background variables and 63 descriptors. Numbers indicate the identifiers of each of the included descriptors for each respective module and serve as a key to the regression coefficients shown in Supplementary Fig. S3. Of originally 285 descriptors, 145 met inclusion criteria (at least 4 observations in each group, lung cancer or no cancer). Additionally-excluded descriptors $(n=82)$ and background variables $(n=9)$ for model finalisation are indicated in Supplementary Table S2. History of chronic obstructive pulmonary disease (COPD) and history of pneumonia, respectively, are physician-confirmed. Bolded descriptors reached significance in terms of regression coefficients and $95 \%$ jack-knifed confidence intervals (ordered by strength of association to lung cancer, see Supplementary Fig. S3). *Indicates variables that had an average regression coefficient with an inverse association to lung cancer $(\mathrm{n}=28)$. 


\begin{tabular}{|l|l|l|l|l|l|l|l|l|}
\hline Model & AUC & AUC2 & $\mathbf{C}$ & $\mathbf{R}^{2} \mathbf{X}$ & $\mathbf{R}^{\mathbf{2}}$ & $\mathbf{Q}^{2}$ & Sens & Spec \\
\hline Full model, 70 variables & 0.767 & 0.695 & 2 & 42.3 & 62.4 & 58.1 & $84.8^{*}$ & $55.6^{*}$ \\
\hline Descriptors only, 63 variables & 0.736 & 0.670 & 2 & 32.7 & 56.0 & 50.1 & 73.9 & 66.7 \\
\hline Background only, 7 variables & 0.652 & 0.568 & 2 & 79.9 & 51.7 & 50.9 & 76.1 & 51.9 \\
\hline
\end{tabular}

Table 3. Lung cancer prediction performance from orthogonal projections to latent structures (OPLS). Table headings: AUC: Area under the receiver operating characteristic (ROC) curve, cross-validation (CV) test set; AUC2: AUC, training set; C: Number of orthogonal components; $\mathbf{R}^{2} \mathbf{X}$ : Percent explained $\mathrm{X}$ variance (for all independent variables); $\mathbf{R}^{2}$ : Percent explained Y variance (lung cancer); $\mathbf{Q}^{2}$ : Cross-validated $\mathbf{R}^{2}$ (CV test set); Sens/Spec: Percent sensitivity and specificity, respectively, of the model in the CV test set, based off the optimal cutoff from the Youden's index. Model abbreviations: Full model: Final model with 70 variables (63 descriptors and seven background variables), built on maximal explained variance $\left(\mathrm{R}^{2}\right.$ and $\left.\mathrm{Q}^{2}\right)$. After initially projecting all 145 descriptors (symptoms/sensations), candidates were then chosen in OPLS by visual inspection of regression coefficients and variable importance for the projection (VIP) values, with sequential removal of descriptors with no model contribution (S1 Table). The seven background variables were selected after demonstrating principal component analysis loadings $>0.1$ and OPLS VIP values $>1$. A full list of the final 70 variables is shown in Table 2. All sensitivity/specificity values are selected from the cutoff with the largest Youden's index. Sensitivity was preferred in this study. *Maximum performance of this model was with Youden's index $=0.426$ favoring specificity: sensitivity $=50 \%$, specificity $=92.6 \%$. Of relevancy for this study, the largest Youden's index tailored for sensitivity (0.402) was selected: sensitivity $=84.8 \%$; specificity $=55.6 \%$.

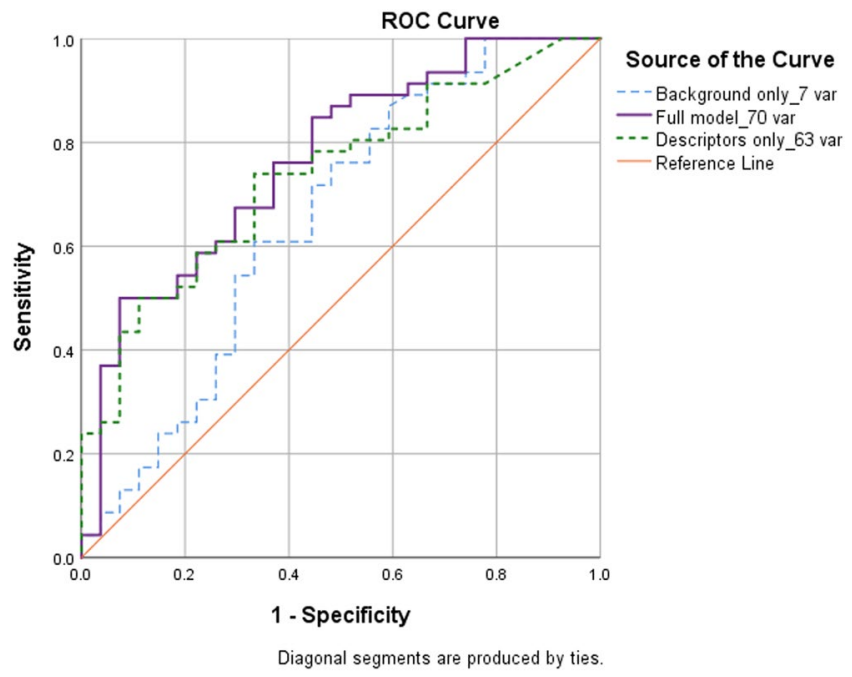

Figure 3. Receiver operating characteristic (ROC) curves for lung cancer prediction performance from orthogonal projections to latent structures (OPLS) modelling. ROC curves of lung cancer prediction performance were calculated from $\mathrm{CV}$ test set lung cancer prediction scores compared to diagnostic outcome (lung cancer or no cancer). Area under the ROC curves are shown in Table 3. For a detailed description of the full model and included variables, see Table 2. Background only_7 var (blue broken line): Seven background variables only. Full model_70 var (purple line): Final model, including 63 descriptors + seven background variables. Descriptors only_63 var (green broken line): 63 descriptors only.

\section{Discussion}

To our knowledge, this is the first study to utilise an interactive e-questionnaire given to individuals referred for LC investigation to comprehensively analyse and identify pre-diagnostic descriptors of symptoms and sensations related to LC. The unique, individualised e-questionnaire that we utilised had a design that allowed us to cover a large number of questions while minimising patient burden. Furthermore, this was combined with a cutting-edge multivariate machine learning analysis of multi-dimensional data to probe how combinations of variables perform in predicting LC. Given the highly variable and heterogeneous symptoms and sensations which were reported, OPLS regression was essential for analysis due to its filtration capability in capturing and centralising predictive variation despite the complexity of our data.

Several cohort risk prediction studies that analysed diffuse general practice medical records ${ }^{17-20}$ and a limited survey $^{22}$ previously identified haemoptysis, dyspnoea, chest pain, cough, weight loss, appetite loss, voice hoarseness, and/or fatigue up to two years before diagnosis as LC risk signs. A recent systematic literature review and meta-analysis highlighted haemoptysis, dyspnoea, cough, and chest pain to be key contributors ${ }^{30}$. Our results are in line with most of these previously-reported early risk factors, including haemoptysis, dyspnoea (breathing worse upon exertion), cough problems (cough that varied over the day), appetite loss, and voice hoarseness; 
A

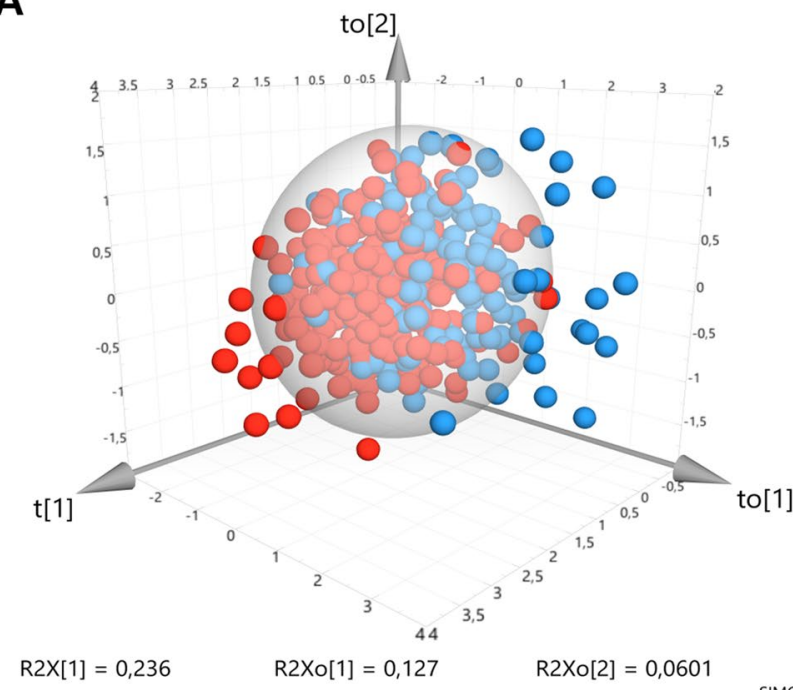

SIMCA 14.1 - 2018-11-20 15:39:43 (UTC+1)

B

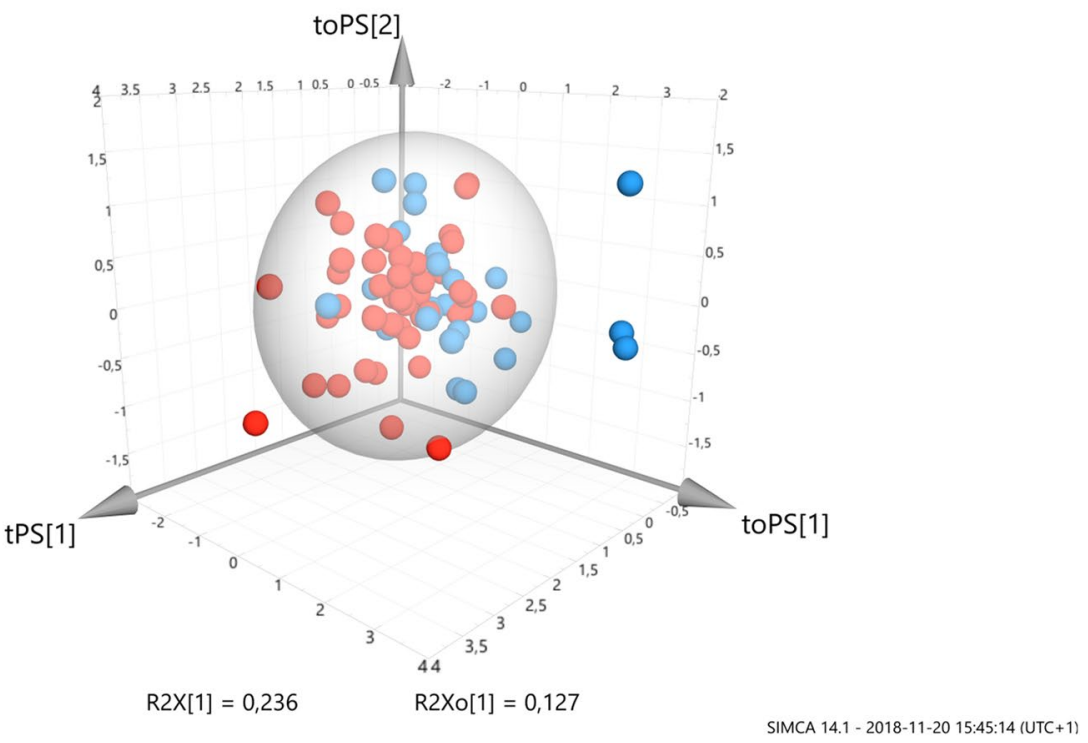

Figure 4. Orthogonal projections to latent structures (OPLS) 3D scores plot. Individual scores for the training set $(\mathbf{A} n=433)$ and predicted scores (PS) for the cross-validated test set $(\mathbf{B} n=73)$ are shown for the final model. All three of the OPLS model components are plotted, including the predictive component $(\mathrm{t}[1])$ and the two orthogonal components (to[1] \& [2]) (total $\mathrm{R}^{2} \mathrm{X}$ variance $=42.3 \%: \mathrm{t}[1]=23.6 \%$, to $[1]=12.7 \%$, to [2] $=6 \%$ ). Predictive explained $\mathrm{R}^{2} \mathrm{Y}$ variance (lung cancer: training set): $62.4 \%$; cross-validated explained $\mathrm{Q}^{2}$ variance (lung cancer: cross-validated test set): $58.1 \%$. A total of 63 descriptors of symptoms and sensations were included together with seven background variables (Table 2). Coloured circles indicate lung cancer (red) or no cancer (blue). Outliers are indicated beyond the 95\% confidence interval ellipse.

and - in addition to active smoking as the most established risk factor - COPD ${ }^{18,19}$ and relatively recent lower/ upper respiratory or non-specific chest infections ${ }^{19}$. On the other hand, through our investigation we identified a plethora of new, early, pre-diagnostic descriptors derived from the patient experience, i.e. early satiety; back pain/aches/discomfort (which could either imply lower or upper back pain; previous models specifically reported only chest pain); having less strength; a heightened sensitivity to different smells; and consistent aches. The identification of these unique descriptors was enabled through the use of an individualised e-questionnaire based on inductive research systematising patients' experiences.

Regarding other risk factors, female sex predicts LC in our results from a Swedish urban setting, which is a disturbing finding. The trend over the past several decades with more women smoking in Sweden points to a need for more cessation programs for women ${ }^{31}$. Finally, we could not confirm that the following previously-reported independent risk signs were predictive of LC, primarily due to exclusion from investigation due to lack of 
observations or not investigating the phenomena, or from a lack of model contribution: thrombocytosis or abnormal spirometry ${ }^{17}$, socioeconomic status ${ }^{18,19}$ or family history of cancer (not investigated, respectively) ${ }^{18}$; other/prior cancer (our endpoint was primary LC only and including this could overfit the model) ${ }^{18}$; and finger clubbing (nail changes) ${ }^{17}$, anaemia ${ }^{18}$ or a chronic cough with chronic phlegm (removed due to lack of model contribution $)^{32}$. We did have information on self-reported weight and weight loss, however, this was missing in a large proportion of patients and we therefore could not draw conclusions other than to state we saw a trend that confirms their inclusion as valuable potential LC predictors as has been previously demonstrated ${ }^{18,19}$.

Two large aforementioned cohort studies have thus far created cross-validated models that include early symptoms with diagnostic performance from patient medical records denoting potential LC risk signs up to two years prior to diagnosis ${ }^{18,19}$. The first model ${ }^{18}$, with haemoptysis, dyspnoea, cough, and appetite loss, had a mean $72 \%$ cross-validated explained variation, 0.92 AUC, and $77.3 \%$ sensitivity for a top $10 \%$ risk score (specificity not reported) (additional background variables included body mass index and weight loss, lower socioeconomic status, ordinal smoking status (cigarettes/day), and, among females, prior cancer). The second model ${ }^{19}$, with haemoptysis, dyspnoea, chest pain, cough, and voice hoarseness, had a 0.88 AUC and a peak sensitivity of $93.98 \%$ vs. $59.67 \%$ specificity in cross-validation (explained variance not reported) (additional background variables included lower socioeconomic status, weight loss, and smoking history (current, past or ordinal by cigarettes/ day)). These metrics can be compared with the performance of our model, with cross-validated explained variance of $58.1 \%$; AUC: 0.767 , and $84.8 \%$ peak sensitivity vs. $55.6 \%$ specificity. While these studies have major strengths in their nationally-representative sample sizes and AUC metrics that outperform our model, they have methodological limitations addressed in our study. In both prior studies, comorbid/previous cancers other than LC were not excluded, leading to a very heterogeneous sample with findings less clinically relevant to primary LC only, in relation to no cancer at all. Additionally, their data derives from general practice record retrieval of a limited set of diffuse symptoms (i.e. cough, chest pain, and dyspnoea), and quality control of descriptors was not possible due to the lack of direct patient interaction. Our findings are thus both robust and novel as we know of no other study using detailed patient-reported descriptors of symptoms and sensations to predict primary LC.

This study has some limitations to consider, including potential patient recall bias due to the retrospective approach. Secondly, predictors could have been made more precise, such as including pack years as opposed to using only current smoking status. Additionally, the predictive value of several rarely-occurring early descriptors could not be determined in our study. Therefore, a larger sample would help in finding the potential importance of these descriptors. With this in mind, while our model accurately predicted LC among a population of at-risk patients who already passed general practice gatekeepers and were subsequently referred to lung specialists, our model also needs to be tested against a more general population to determine its validity as a potential tool to help flag patients early for diagnostic workup.

The present study was able to identify unique early patient-reported descriptors predictive of LC among a vast array of 285 descriptors investigated through an advanced modelling approach from data collected with an interactive tablet questionnaire tailored for usability. While several LC descriptors identified by us have been previously described, our unique approach allowed identification of novel descriptive indicators of LC risk that can be integrated into a simplified questionnaire in future LC investigation. Signs of early satiety before diagnosis and treatment, for example, was a major early LC predictor in the current study that has, to our knowledge, not been identified before. Our specific, in-depth and complex investigation allowed for key descriptors to surface, and such an approach requires an advanced method like OPLS to handle the magnitude of variables by projection instead of being directly influenced by- or needing to control for the amount of variables ${ }^{23-26}$. As a potential tool for use in clinical practice, the 70 variables identified may at a later stage be administered as a questionnaire to individuals exhibiting respiratory-related distress, whereby the resulting OPLS risk-prediction score may be used to flag patients for specialized diagnostic workup. Furthermore, PEX-LC could be tested to tackle the large false positive rate problem in conjunction with CT-based LC screening to prioritize patient selection from large risk-group populations.

\section{Conclusions}

This is a first step towards identifying optimal patient-reported predictive markers for LC, and combining these with relevant biological markers may represent the most promising means to reduce LC mortality apart from smoking cessation. The results from this advanced modelling approach applied on early symptoms and sensations derived from an interactive e-questionnaire may lead to a tool for referral and LC diagnostic decision-making, thus potentially facilitating a more timely diagnosis and improving LC survival.

\section{Data availability}

Data cannot be shared publicly due to protecting the privacy of the patients who agreed to participate in the study. The anonymised dataset utilised for analyses carried out for the current study is available from the corresponding author on reasonable request.

Received: 12 May 2019; Accepted: 23 October 2019;

Published online: 11 November 2019

\section{References}

1. Alberg, A. J., Brock, M. V., Ford, J. G., Samet, J. M. \& Spivack, S. D. Epidemiology of lung cancer: Diagnosis and management of lung cancer, 3rd ed: American College of Chest Physicians evidence-based clinical practice guidelines. Chest 143, e1S-e29S, https://doi. org/10.1378/chest.12-2345 (2013).

2. Molina, J. R., Yang, P., Cassivi, S. D., Schild, S. E. \& Adjei, A. A. Non-small cell lung cancer: epidemiology, risk factors, treatment, and survivorship. Mayo Clin Proc 83, 584-594, https://doi.org/10.4065/83.5.584 (2008).

3. Ferlay, J. et al. Cancer incidence and mortality worldwide: sources, methods and major patterns in GLOBOCAN 2012. Int J Cancer 136, E359-386, https://doi.org/10.1002/ijc.29210 (2015). 
4. Corner, J., Hopkinson, J., Fitzsimmons, D., Barclay, S. \& Muers, M. Is late diagnosis of lung cancer inevitable? Interview study of patients' recollections of symptoms before diagnosis. Thorax 60, 314-319, https://doi.org/10.1136/thx.2004.029264 (2005).

5. Corner, J., Hopkinson, J. \& Roffe, L. Experience of health changes and reasons for delay in seeking care: a UK study of the months prior to the diagnosis of lung cancer. Soc Sci Med 62, 1381-1391, https://doi.org/10.1016/j.socscimed.2005.08.012 (2006).

6. Spitz, M. R. et al. A risk model for prediction of lung cancer. J Natl Cancer Inst 99, 715-726, https://doi.org/10.1093/jnci/djk153 (2007).

7. Cassidy, A. et al. The LLP risk model: an individual risk prediction model for lung cancer. Br J Cancer 98, 270-276, https://doi. org/10.1038/sj.bjc.6604158 (2008).

8. Brindle, L., Pope, C., Corner, J., Leydon, G. \& Banerjee, A. Eliciting symptoms interpreted as normal by patients with early-stage lung cancer: could GP elicitation of normalised symptoms reduce delay in diagnosis? Cross-sectional interview study. BMJ Open 2, https://doi.org/10.1136/bmjopen-2012-001977 (2012).

9. Mitchell, E. D., Rubin, G. \& Macleod, U. Understanding diagnosis of lung cancer in primary care: qualitative synthesis of significant event audit reports. Br J Gen Pract 63, e37-46, https://doi.org/10.3399/bjgp13X660760 (2013).

10. Wagland, R. et al. Facilitating early diagnosis of lung cancer amongst primary care patients: The views of GPs. Eur J Cancer Care (Engl) 26, https://doi.org/10.1111/ecc.12704 (2017).

11. Oudkerk, M. et al. European position statement on lung cancer screening. Lancet Oncol 18, e754-e766, https://doi.org/10.1016/ S1470-2045(17)30861-6 (2017)

12. Integrative Analysis of Lung Cancer Etiology and Risk (INTEGRAL) Consortium for Early Detection of Lung Cancer. et al. Assessment of Lung Cancer Risk on the Basis of a Biomarker Panel of Circulating Proteins. JAMA Oncol 4, e182078, https://doi. org/10.1001/jamaoncol.2018.2078 (2018).

13. van Klaveren, R. J. et al. Management of lung nodules detected by volume CT scanning. N Engl J Med 361, 2221-2229, https://doi. org/10.1056/NEJMoa0906085 (2009).

14. National Lung Screening Trial Research Team. et al. Reduced lung-cancer mortality with low-dose computed tomographic screening. N Engl J Med 365, 395-409, https://doi.org/10.1056/NEJMoa1102873 (2011).

15. Ru Zhao, Y. et al. NELSON lung cancer screening study. Cancer Imaging 11 Spec No A, S79-84, https://doi.org/10.1102/14707330.2011 .9020 (2011).

16. Noone, A. M. et al. SEER Cancer Statistics Review, 1975-2015 (National Cancer Institute, Bethesda, MD, 2017).

17. Hamilton, W., Peters, T. J., Round, A. \& Sharp, D. What are the clinical features of lung cancer before the diagnosis is made? A population based case-control study. Thorax 60, 1059-1065, https://doi.org/10.1136/thx.2005.045880 (2005).

18. Hippisley-Cox, J. \& Coupland, C. Identifying patients with suspected lung cancer in primary care: derivation and validation of an algorithm. Br J Gen Pract 61, e715-723, https://doi.org/10.3399/bjgp11X606627 (2011).

19. Iyen-Omofoman, B., Tata, L. J., Baldwin, D. R., Smith, C. J. \& Hubbard, R. B. Using socio-demographic and early clinical features in general practice to identify people with lung cancer earlier. Thorax 68, 451-459, https://doi.org/10.1136/thoraxjnl-2012-202348 (2013).

20. Jones, R., Latinovic, R., Charlton, J. \& Gulliford, M. C. Alarm symptoms in early diagnosis of cancer in primary care: cohort study using General Practice Research Database. BMJ 334, 1040, https://doi.org/10.1136/bmj.39171.637106.AE (2007).

21. Walter, F. M. et al. Symptoms and other factors associated with time to diagnosis and stage of lung cancer: a prospective cohort study. Br J Cancer 112(Suppl 1), S6-13, https://doi.org/10.1038/bjc.2015.30 (2015).

22. Lever, J., Krzywinski, M. \& Atman, N. Points of significance: principal component analysis. Nat Methods 14, 641-642, https://doi. org/10.1038/nmeth.4346 (2017).

23. Trygg, J. \& Wold, S. Orthogonal projections to latent structures (O-PLS). J. Chemometrics 16, 119-128, https://doi.org/10.1002/ cem.695 (2002).

24. Verron, T., Sabatier, R. \& Joffre, R. Some theoretical properties of the O-PLS method. J. Chemometrics 18, 62-68, https://doi. org/10.1002/cem.847 (2004).

25. Trygg, J. Prediction and spectral profile estimation in multivariate calibration. J. Chemometrics 18, 166-172, https://doi.org/10.1002/ cem.860 (2004).

26. Whelehan, O. P., Earll, M. R., Johansson, E., Toft, M. \& Eriksson, L. Detection of ovarian cancer using chemometric analysis of proteomic profiles. Chemometrics and Intelligent Laboratory Systems 84, 82-87 (2006).

27. Triba, M. N. et al. PLS/OPLS models in metabolomics: the impact of permutation of dataset rows on the K-fold cross-validation quality parameters. Mol Biosyst 11, 13-19, https://doi.org/10.1039/c4mb00414k (2015).

28. Eriksson, L. et al. Multi- and Megavariate Data Analysis, Part I: Basic Principles and Applications. (Umetrics AB, 2006).

29. Hosmer, D. W. \& Lemeshow, S. Applied Logistic Regression. Second edn, 160-62 (John Wiley \& Sons, Inc., 2005).

30. Okoli, G. N., Kostopoulou, O. \& Delaney, B. C. Is symptom-based diagnosis of lung cancer possible? A systematic review and metaanalysis of symptomatic lung cancer prior to diagnosis for comparison with real-time data from routine general practice. PLoS One 13, e0207686, https://doi.org/10.1371/journal.pone.0207686 (2018).

31. Koyi, H., Hillerdal, G. \& Branden, E. A prospective study of a total material of lung cancer from a county in Sweden 1997-1999: gender, symptoms, type, stage, and smoking habits. Lung Cancer 36, 9-14 (2002).

32. Kubik, A. K., Zatloukal, P., Tomasek, L. \& Petruzelka, L. Lung cancer risk among Czech women: a case-control study. Prev Med 34, 436-444, https://doi.org/10.1006/pmed.2001.1002 (2002).

\section{Acknowledgements}

This study has received research support from The Vårdal Foundation (ref no 2014-0044), Swedish Research Council (ref no 2016-01712), and the Strategic Research Area Health Care Science (SFO-V, ref no 2-2764/2018). The funding sources had no role in study design; neither in the collection, analysis, and interpretation of data or writing of the report; nor in the decision to prepare and submit the paper for publication. Open access funding provided by Karolinska Institute.

\section{Author contributions}

A.L. is the first author and wrote the majority of the manuscript, created all tables and figures, and conceptualised and performed all analyses and the literature search; M.P. conceptualised the analysis and supervised interpretation of data, writing and analyses; B.M.B. contributed to data collection, interpretation, and writing; J.F. contributed to data interpretation and guidance on data analysis; K.K. ensured the study could be carried out at the study sites; M.O. coordinated on-site data collection; R.H. contributed to data interpretation and discussion; J.L. supervised data interpretation and analyses; C.T. is a senior author and initial principal investigator who designed PEX-LC, conceptualised the analysis, and supervised study conduction, data interpretation, and writing; L.E.E. is the corresponding senior author, principal investigator and designer of PEX-LC, conceptualised the analysis, and supervised study conduction, data interpretation, analyses, and writing. All authors contributed to the writing and discussion of the manuscript and meet all criteria of the ICMJE criteria for authorship. 


\section{Competing interests}

The authors declare no competing interests.

\section{Additional information}

Supplementary information is available for this paper at https://doi.org/10.1038/s41598-019-52915-x.

Correspondence and requests for materials should be addressed to L.E.E.

Reprints and permissions information is available at www.nature.com/reprints.

Publisher's note Springer Nature remains neutral with regard to jurisdictional claims in published maps and institutional affiliations.

(c) (i) Open Access This article is licensed under a Creative Commons Attribution 4.0 International License, which permits use, sharing, adaptation, distribution and reproduction in any medium or format, as long as you give appropriate credit to the original author(s) and the source, provide a link to the Creative Commons license, and indicate if changes were made. The images or other third party material in this article are included in the article's Creative Commons license, unless indicated otherwise in a credit line to the material. If material is not included in the article's Creative Commons license and your intended use is not permitted by statutory regulation or exceeds the permitted use, you will need to obtain permission directly from the copyright holder. To view a copy of this license, visit http://creativecommons.org/licenses/by/4.0/.

(C) The Author(s) 2019 